\title{
Sky brightness measurements for different environmental conditions by the example of St. Petersburg
}

\author{
Svetlana Kolgushkina ${ }^{1}$ \\ ${ }^{1}$ ITMO University, School of Photonics, Faculty of Laser and Light Engineering, Department of \\ Creative Lighting Design, St. Petersburg, Russia
}

\begin{abstract}
St. Petersburg is unique place in Russia with an environmental phenomenon called "white nights" during summertime: sky brightness levels are influenced mostly by environmental conditions. Sky conditions during the winter are opposite: light emission is mostly caused by the anthropogenic factors. A series of experiments were conducted between May and December 2017 using a Sky Quality Meter (SQM-LU-DL), a night sky brightness photometer, to understand the differences between sky brightness levels for different environmental conditions and seasonal variations. Sky brightness distinction between the city center and $20 \mathrm{~km}$ distance were estimated for clear sky conditions.
\end{abstract}

\section{Introduction}

Lighting design solutions in 21 st century should be carried out with considering environmental conditions of place in order to improve its uniqueness. Sky brightness estimation during the night could show the distinctive features of different cities depending on locations. For example, near the equator duration of the night is about 12 hours both in winter and summer with insignificant duration of twilight. For the places near the North, at the same time, length of night strongly depends on the season. Transient moment between day and night is extended in time. Environmental influence on sky brightness is considerable during clear nights while artificial light impact more corresponds to cloudy weather conditions.

For field measurements documenting the evolution of sky brightness, different types of night sky brightness photometers, such as the Unihedron Sky Quality Meter, are widely used. $[1,2]$ The SQM-LU-DL, a pocket-sized night sky brightness photometer with a data-logging system inside, was used to study St. Petersburg's artificial light emission. According to a published report of the relationship between the SQM photometrical system and the main systems used in light pollution studies, correction factors to CIE photopic and CIE scotopic were taken into account. $[3,4]$

\footnotetext{
${ }^{1}$ Corresponding author: svkolgushkina@corp.ifmo.ru
} 


\section{Field of study}

The city of St. Petersburg is a very interesting example for corresponding research for a number of reasons: there are no laws, codes and standards in the Russian Federation for artificial light emission regulations and natural sky conditions change very significantly during the year: from high levels during summer time with a short period of dusk to a short daytime period during winter.

Changes in daylight hours during the year are very significant: on 22 December, the duration of the day in 2017 was $5 \mathrm{~h} 53 \mathrm{~m}$ while on 22 June it was $18 \mathrm{~h} 50 \mathrm{~m}$. The dynamics of daylight changes from May to December are shown in Figure 1.

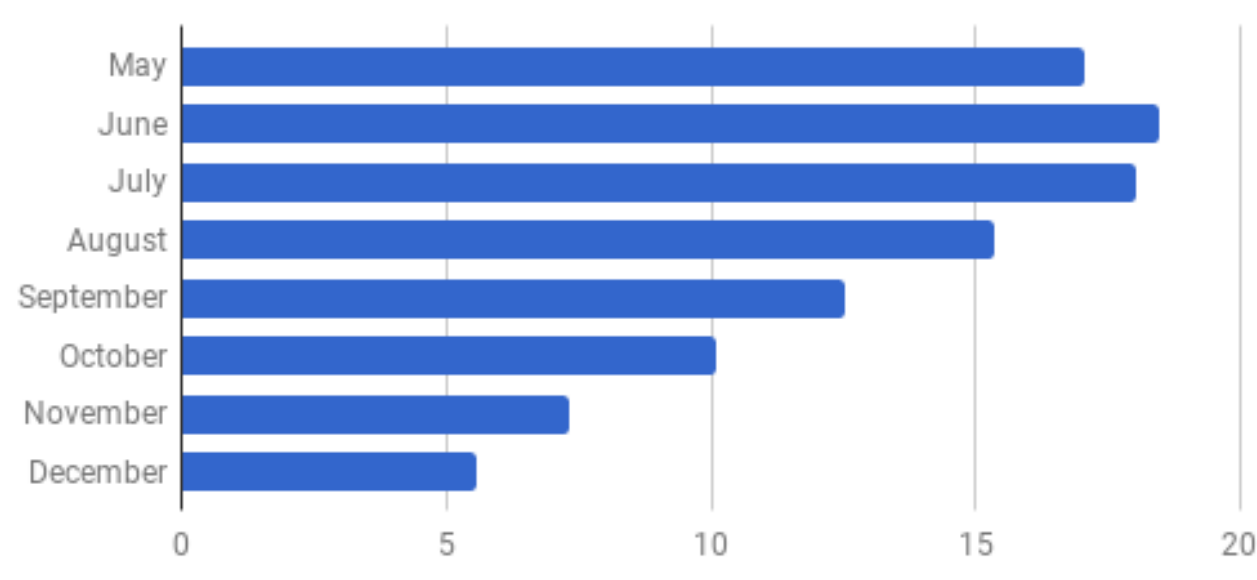

Figure 1. Dynamics of daylight hours during the period May - December 2017.

Night sky brightness is influenced by anthropogenic sky glow, which depends on weather conditions, background sky luminance, the position of the sun (indirectly scattered sunlight), and the phases of the moon. During the summer in St. Petersburg, luminance levels over the night sky are influenced mostly by environmental conditions, while during the winter, sky brightness levels are caused by artificial light emission on the molecules of water and aerosol. The quantity of overcast nights during December is 26 out of 31 days, while during June, it is 11 out of 31 days, as shown in Figure 2. 


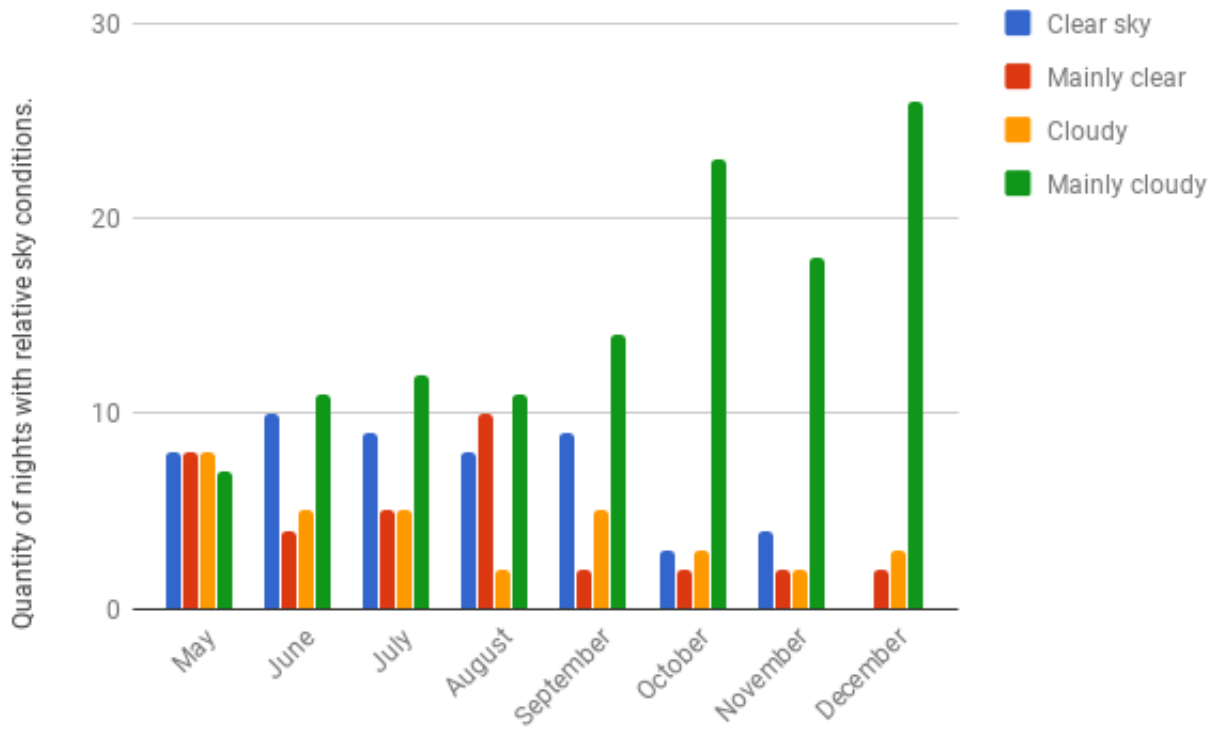

Figure 2. Quantity of nights with relative sky conditions.

The perception of sky conditions from the observer's perspective changes from summer to winter, as shown in Figure 3 and Figure 4.

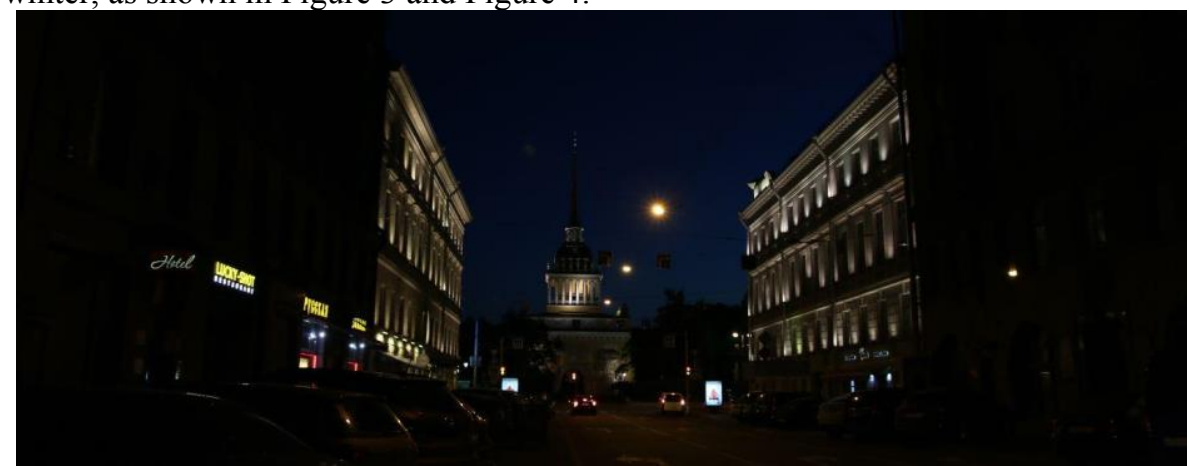

Figure 3. Sky perception from the position of observer in June during "white nights".

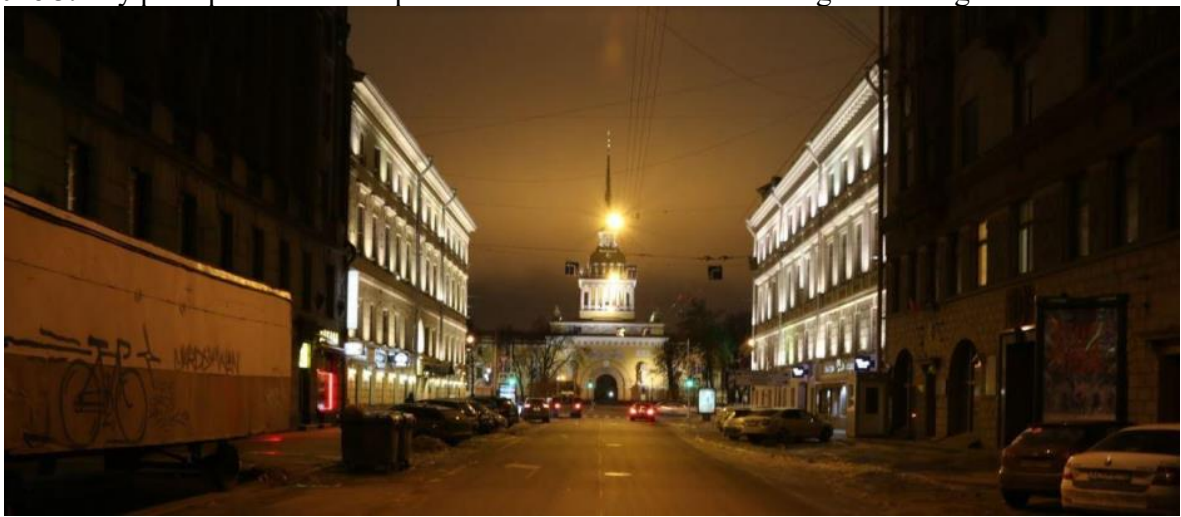

Figure 4. Sky perception from the position of observer in December. 


\section{Methodology}

For artificial light emission analysis there were used principles of modeling artificial sky brightness formed by Garstang in his model. 18 districts of St. Petersburg were considered separately.

In order to estimate sky brightness differences through the seasons series of field measurements were obtained using the Unihedron brightness photometer SQM-LU-DL aimed at quantitatively comparing and monitoring sky brightness through the night and nightto-night. The SQM-LU-DL device provides readings in magnitudes per square arcsecond $\frac{\mathrm{mag}}{\operatorname{arcsec}^{2}}$, a logarithmic measurement mostly used by astronomers to measure sky brightness. A decrease of magnitude value means an increase in the amount of light coming from a given patch of sky. A change of $5 \frac{\mathrm{mag}}{\operatorname{arcsec}^{2}}$ means a 100 time enlargement of the sky brightness. Each SQM-LU-DL is factory-calibrated. The absolute precision of each device is believed to be $\pm 0.10 \frac{\mathrm{mag}}{\operatorname{arcsec}^{2}}$. The operating temperature range is -40 to $85 C^{0}$.

A series of measurements were taken at the central side of the city between May December 2017 at midnight for different weather conditions and moonphase at zenith angle zero in order to avoid influence of existing outdoor lighting fixtures. For each sky brightness analysis there were made series of measurements with data-logging every 5 seconds within 2 minutes which then were averaged. To compare sky brightness levels in the central part of the city with amount of light emission outside the city, a series of experiments were made 20 kilometres from the centre of the city. SQM-LU-DL was equipped with the lens at half width half maximum (HWHM) of the angular sensitivity $\left(\sim 10^{\circ}\right)$. The block diagram of the device is shown Figure 5 and Figure 6.
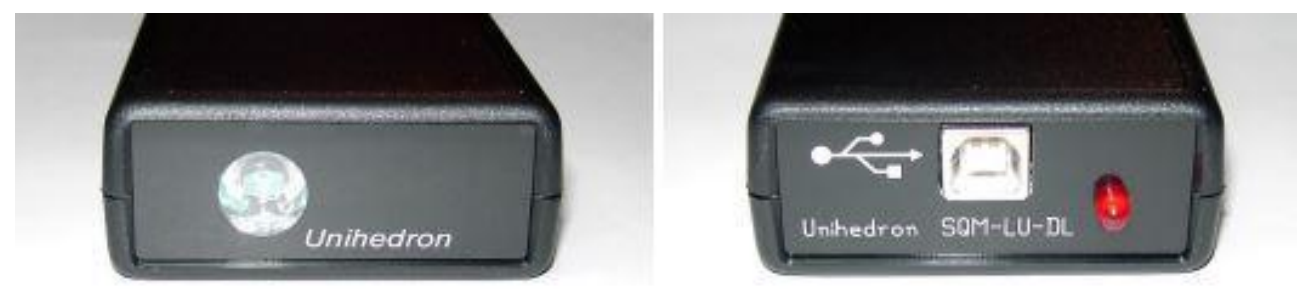

Figure 5. SQM-LU-DL device with a data-logging system inside.

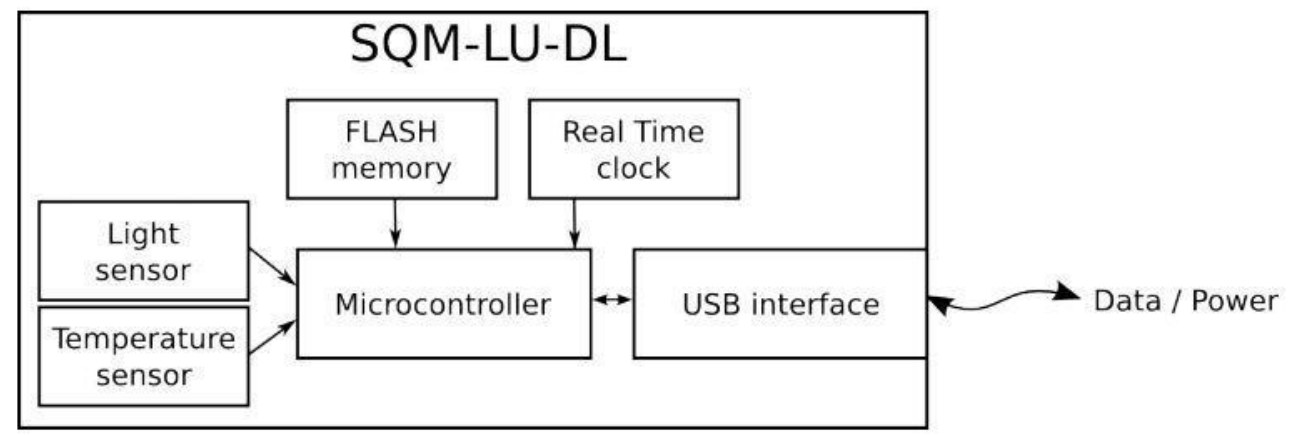

Figure 6. SQM-LU-DL device block diagram.

To convert the SQM Magnitudes per square arcsecond readings to $\mathrm{cd} / \mathrm{m}^{2}$ the following equation (1) was used in accordance with manufacturer's data: 


$$
\left[c d / m^{2}\right]=10,8 \times 10^{4} \times 10^{\left(-0,4 \times\left[\mathrm{mag}_{\operatorname{arcsec}}{ }^{2}\right]\right)}
$$

(1)

According to the calculations made by $\mathrm{P}$. Cinzano aimed at evaluating the relationship between the SQM photometric band and the CIE photometric system, the Sky Quality Meter is properly calibrated over Illuminant A. [3] P. Cinzano calculated spectral mismatch correction factors for the CIE photopic and scotopic responses as shown in Table 1. During the experiment, all of this information was taken into account.

Table 1. Spectral mismatch correction factors for CIE photopic and scotopic responses.

\begin{tabular}{|c|c|c|c|c|c|c|}
\hline $\begin{array}{c}\text { Light } \\
\text { source }\end{array}$ & HPS lamp & HPL lamp & $\begin{array}{c}\text { Illuminant } \\
\text { A }\end{array}$ & $\begin{array}{c}\text { Sky } \\
\text { natural }\end{array}$ & $\begin{array}{c}\text { Sky } \\
\text { polluted }\end{array}$ & $\begin{array}{l}\text { Moon ab. } \\
\text { atm }\end{array}$ \\
\hline Photopic & 0,78 & 1,02 & 1,00 & 0,96 & 0,85 & 1,20 \\
\hline Scotopic & 1,70 & 1,22 & 1,00 & 0,79 & 1,37 & 0,79 \\
\hline
\end{tabular}

\section{Results}

During the on-field research there've been analysed night sky brightness depending both the lunar phase and artificial light emission for clear sky and cloudy weather conditions depending on the night. All the data in Magnitudes per square arcsecond were converted to the lighting metrics system. Data averaged for the midnights with different sky conditions and moon phases during the experiment are shown on the Figures 8-9.

The most influencing factor of artificial light emission is cloudiness. In December there were no clear sky nights and the level of brightness tends to have close values with the luminance on the roads. Moonlight influence is higher during clear nights than during cloudy nights. If there were no artificial light over the city, brightness of the overcast night would be lower, than the clear. But according to the measurements, sky during the overcast winter is brighter than during the shortest night of the year (June 22). Clear sky conditions with $0,5 \%$ Moon correspond to Minimum values during the month while overcasted sky with $95 \%$ Moon - to Maximum values. The dynamics of changes from May to December are shown in Figure 7 and Figure 8.

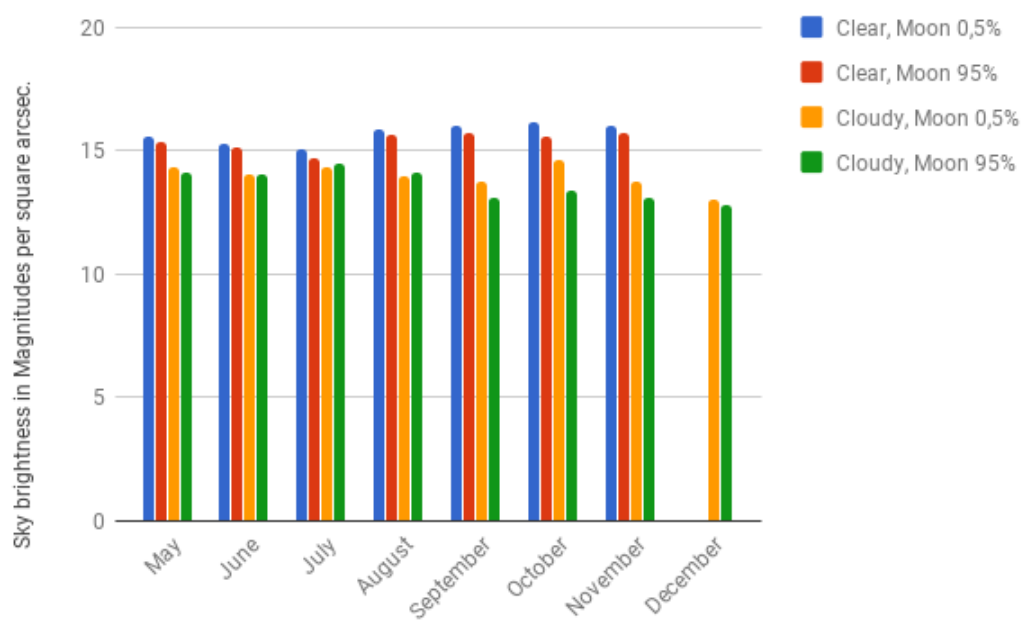


Figure 7. Sky brightness level depending on the month and sky conditions in Magnitudes per square arcsec at midnight between May - December 2017.

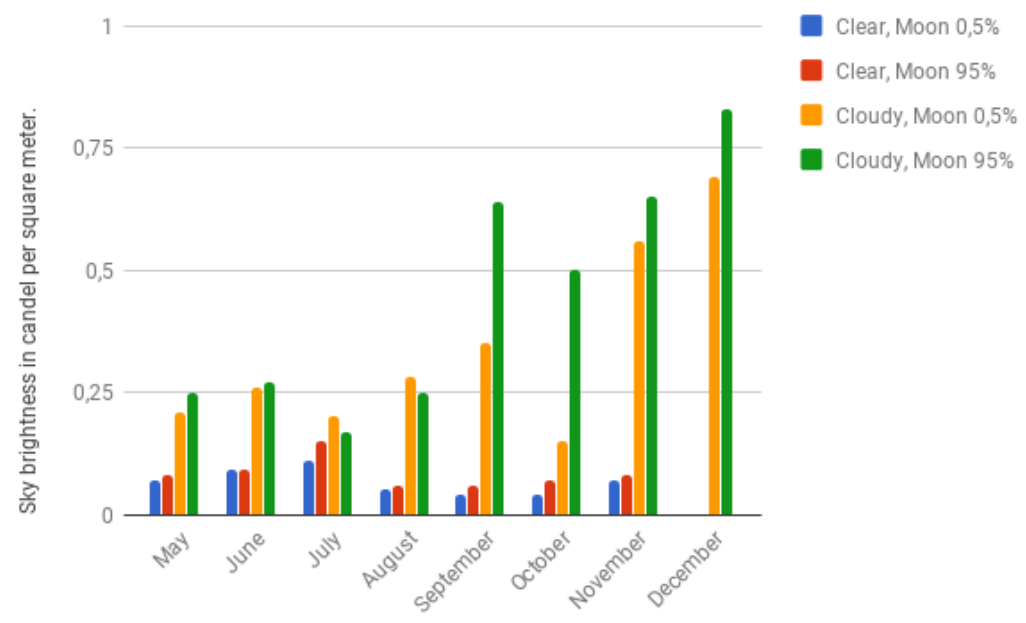

Figure 8. Sky brightness levels depending on the month and sky conditions in candela per square meter.

Comparing the measurements for clear sky conditions in the city center to the measurements for on 20 kilometers distance from the central side differences in values are shown in Figure 9.

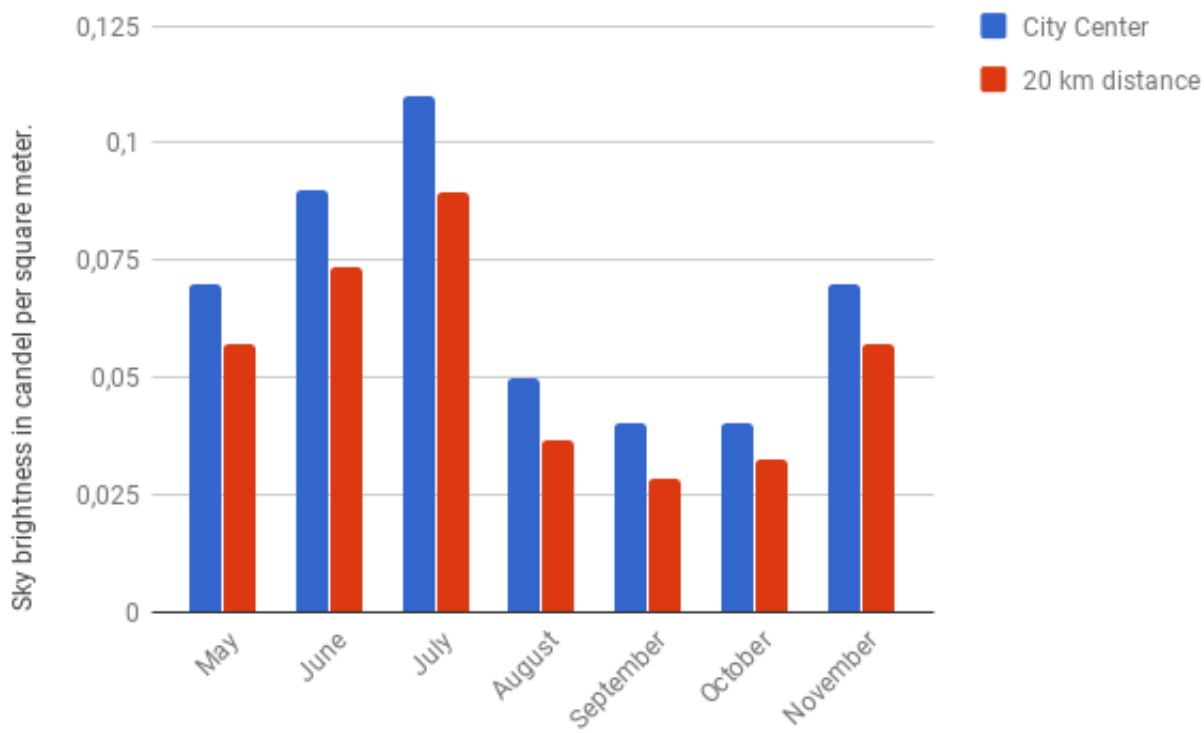

Figure 9. Sky brightness for clear sky conditions in the city center and on $20 \mathrm{~km}$ distance without moonlight.

The differences in sky brightness values between the city center and $20 \mathrm{~km}$ distance for clear sky conditions are about $17-21 \%$ depending on the month. 
Statistical analysis of measurements for midnight sky brightness between May December 2017 refers to the following results: during clear and moonless sky conditions night sky brightness at St. Petersburg reaches values during summer 15,03-15,55 $\frac{\mathrm{mag}}{\operatorname{arcsec}^{2}}$ in transition to lighting metrics $0,07-0,11 \mathrm{~cd} / \mathrm{m}^{2}$.

During the winter values for clear sky are close to $15,45-16,1 \frac{\operatorname{mag}}{\operatorname{arcsec}^{2}}$ in transition to lighting metrics $0,04-0,07 \mathrm{~cd} / \mathrm{m}^{2}$. Overcast sky is mainly caused by the scattering process on aerosols and moleculas and sky brightness levels are always higher than during the clear sky nights and reaches the values $14,28-12,82 \frac{\mathrm{mag}}{\operatorname{arcsec}^{2}}\left(0,21-0,83 \mathrm{~cd} / \mathrm{m}^{2}\right)$.

\section{Conclusions}

Sky brightness during the night is an important link among environmental aspects and perception from perspective of observer. Sky glow is approaching by value to luminance levels on the roads' surfaces with significant impact on adaptation and could be considered as a factor influencing on perception of contrast.

Brightness levels, as litmus paper for lighting design needs, could help to evaluate both natural conditions of night sky and contribution to sky glow of existing lighting in order to identify:

- environmental identity of place

- potential ways for further development of lighting design in the cityscape

All of these aspects have clear prospects in lighting design. For example, natural sky estimation could be used for development of integrated lighting control systems with respect to environmental changes. Corresponding solutions are potentially interesting for St. Petersburg and the other North cities with similar environmental phenomenons.

Considering the impact of existing lighting to sky glow, critical values could be evaluated. Then, if sky brightness is higher than critical, special codes and standards recusing sky glow should be developed. This approach is relevant for a large number of cities, especially metropolises, with a significant leap in quantity of lighting in recent years.

\section{References}

1. Birriel, J. Documenting Local Night Sky Brightness Using Sky Quality Meters: An Interdisciplinary College Capstone Project and a First Step Toward Reducing Light Pollution Publication of JAAVSO Volume 38, 2010, 132-138

2. Benn, C. R., \& Ellison, S. L. Brightness of the night sky over La Palma, New Astronomy Reviews, 1998, 503-507.

3. Cinzano P. Night sky photometry with sky quality meter. Internal Report n. 9, v.1.4; Istituto di Scienza e Tecnologia dell Inquinamento Luminoso (ISTIL); 2005.

4. Lolkema, D., Haaima, M., den Outer, P., \& Spoelstra, H. 2010, Effects of atmospheric conditions on night sky brightness, Tech. rep., National Institute for Public Health and the Environment, RIVM Report 680151002, 2010 